mer's disease brain. Q. J. Med.; in press.

10. Jeste, D. V.; Lohr, J. B.; Goodwin, F. K. Anatomical studies of major affective disorders. A review and suggestions for further research. Br. J. Psychiatry 153:444 459; 1988.

11. Korey, S. R.; Scheinberg, L.; Terry, R.; Stein, A. Studies in presenile dementia. Trans. Am. Neurol. Assoc. 86:99-102; 1961

12. Marx, J. L. Brain protein yields clues to Alzheimer's disease. Science 243:1664-1666; 1989.

13. Mesulam, M. M.; Geula, C. Acetylcholinesterase-rich pyramidal neurons in the human neocortex and hippocampus. Absence at birth, development during the life span, and dissolution in Alzheimer's disease. Ann. Neurol. 24:765-773; 1988.

14. Procter, A. W.; Bowen, D. M. Ageing, the cerebral neocortex and psychiatric disorder. In: Davies, P.; Finch, C. E., eds. Molecular neuropathology of ageing. Banbury Report 27. Cold Spring Harbor
Laboratory; 1987:3-20.

15. Procter, A. W.; Wong, E. H. F.; Stratmann, G. C.; Lowe, S. I Bowen, D. M. Reduced glycine stimulation of [ $\left.{ }^{3} \mathrm{H}\right] \mathrm{MK} 801$ binding in Alzheimer's disease. J. Neurochem. 53:698-704; 1989.

16. Procter, A. W.; Stirling, J. H.; Stratmann, G. C.; Cross, A. J.: Bowen, D. M. Loss of glycine-dependent radioligand binding to the $\mathrm{N}$-methyl-D-aspartate-phencyclidine receptor complex in patients with Alzheimer's disease. Neurosci. Lett. 101:62-66;1989.

17. Steele, J. E.: Palmer, A. M.; Lowe, S. L.; Bowen, D. M. The influence of tetrahydro-9-aminoacridine on excitatory amino acid neurotransmission in vivo and in vitro. Br. J. Pharmacol. 96:353P; 1989

18. Wilkins, R. H.; Brody, I. A. Alzheimer's disease. Arch. Neurol. 21:109-110; 1969 .

\title{
Authors' Response to Commentaries
}

\section{J. TIMOTHY GREENAMYRE AND ANNE B. YOUNG ${ }^{1}$}

\author{
Department of Neurology, University of Michigan \\ Neuroscience Laboratory Building, 1103 East Huron, Ann Arbor, MI 48104-1687
}

WE agree with Gary Gibson that the "test of a good hypothesis is not whether it is right or wrong, but that it stimulates productive and revealing experiments." In our review, we presented two distinct hypotheses. First, we summarized evidence suggesting both pre- and postsynaptic disruption of excitatory amino acid (EAA) pathways in Alzheimer's disease and we have hypothesized that these abnormalities may account for some of the clinical manifestations of this disease. Second, we have proposed that EAA, by virtue of their excitotoxic properties, might play a role in the pathogenesis of Alzheimer's disease. Our review was intentionally provocative and the commentators have raised a number of interesting points, some of which we will address below.

Little disagreement exists that EAA-utilizing neurons are affected in Alzheimer's disease. Determination of the extent of EAA system damage in Alzheimer's disease is limited by the lack of an adequate, stable marker for EAA neurons in postmortem brain. As Dr. McGeer indicates in her commentary, phosphateactivated glutaminase is a potential marker for EAA neurons, but its specificity is uncertain. If glutaminase proves to be a reliable marker for EAA neurons, the much greater loss of glutaminase in Alzheimer's disease than of choline acetyltransferase will be of immense interest. These results could indicate an early, severe loss of EAA neurons in Alzheimer's disease.

We agree with Cotman et al. that NMDA receptor loss is not widespread in mild to moderate Alzheimer's disease and that receptor loss in advanced Alzheimer's disease is likely related, in part, to cell loss. We disagree, however, with their interpretation of the current literature regarding NMDA/PCP receptor losses in Alzheimer's disease and with the conclusion "it now appears that NMDA receptor number is relatively maintained in cases of moderate cell loss in AD so that the cellular density of NMDA receptors may actually increase ...." This conclusion is perplexing since they report an average decline of $40 \%$ in TCP binding in CAl of Alzheimer's hippocampus (8) which was statistically significant $(p<0.013)$, despite their implication to the contrary in the commentary. A critical examination of the literature would indicate that no published study has investigated the relationship of NMDA receptor number to neuronal cell counts or the integrity of neuronal processes in Alzheimer's disease. Only reports of NMDA receptor binding relative to binding to other receptors have appeared $(3,4)$. These studies, indeed, indicate that NMDA/PCP receptors are more reduced than other receptors in CA1 in Alzheimer's hippocampus. Considerably more detailed postmortem information must be obtained before drawing such strong, albeit intriguing, conclusions based on limited data. In addition, ascribing hippocampal cell death in Alzheimer's disease to agonal hypoxia appears unwarranted until some additional supportive data are obtained. We disagree also with Cotman et al.'s interpretation of our previous studies of $\left[{ }^{3} \mathrm{H}\right] \mathrm{glutamate}$ binding in calcium and chloride (4). We have studied in detail the properties of the calcium/chloride-dependent $\left[{ }^{3} \mathrm{H}\right]$ glutamate binding site in autoradiographic binding studies $(1,2)$. (Cotman and colleagues have studied ion-dependent binding in homogenates and this homogenate binding has very different properties than the autoradiographic binding sites we have described.) The autoradiographic site is located postsynaptically on neurons, not glia, is unlikely to be a transport site and probably represents binding to both the ion channel-linked quisqualate receptor and the quisqualate-linked metabotropic receptor $(1,2)$. We have also confirmed the large loss of NMDA receptors $(>80 \%)$ in hippocampus of severe $\mathrm{AD}$ cases by examining NMDA displaceable binding directly in the original series of cases $[(3) ;$ J. B. Penney et al., submitted].

Dr. McGeer suggests that "measurements of the integrity of glycine and PCP modulation" of the NMDA receptor complex "might be more revealing than simple measurements of NMDA binding density." This is apparently true, Autoradiographic studies from our lab, as well as from Carl Cotman's lab, have shown 
substantial and significant losses $(40-55 \% ; p<0.02)$ of PCP sites in CA1 of Alzheimer's disease hippocampus $(6,8)$. In contrast, homogenate studies of binding to the PCP site have shown no changes in PCP receptors in the hippocampus of Alzheimer's disease patients $(9,11)$. What is the difference between autoradiographic and homogenate binding assays? Aside from the enhanced anatomical resolution obtainable with autoradiography, the residual ambient glycine levels may be critical. It is extremely difficult to remove all of the endogenous glycine from slidemounted tissue sections, whereas it is much easier to remove glycine in homogenate membrane preparations. In our autoradiographic assay, adding exogenous glycine to the assay results in a variable, modest $(10-40 \%)$ increase in $\left[{ }^{3} \mathrm{H}\right] \mathrm{MK}-801$ binding to the PCP site. When the tissue sections are washed more rigorously, glycine induces a larger, more consistent increase in [ $\left.{ }^{3} \mathrm{H}\right] \mathrm{MK}-801$ binding (S. Y. Sakurai et al., unpublished results). As David Bowen and colleagues report in their commentary, glycine can induce a $300 \%$ increase in $\left[{ }^{3} \mathrm{H}\right] \mathrm{MK}-801$ binding in well-washed membrane preparations from human cortical material. In Alzheimer's disease cortex, Bowen and colleagues observed a marked reduction in this glycine-induced increase in $\left[{ }^{3} \mathrm{H}\right] \mathrm{MK}-801$ binding. Thus, glycine modulation of the NMDA receptor complex appears to be altered in Alzheimer's disease; PCP sites themselves may not be as severely affected. This finding may help to explain the discrepancy between PCP receptor binding in Alzheimer's disease in autoradiographic versus homogenate preparations. In autoradiographic assays, where there is residual endogenous glycine, glycine enhancement of PCP receptor binding is substantial and, therefore, autoradiographic PCP receptor binding is, in part, a measure of glycine modulation. However, in well-washed homogenate preparations in which glycine has been effectively removed, there will be little if any glycine enhancement and therefore less apparent change in PCP receptor binding in Alzheimer's disease.

As we pointed out in our review, EAA mechanisms may be a secondary cause of neuronal damage in Alzheimer's disease because neurons which have been compromised by some other pathological process become more susceptible to NMDA neurotoxicity. Drs. Lee and Sapolsky et al. both emphasize that multiple factors rather than a unitary process are likely to be responsible for the selective neuronal vulnerability observed in Alzheimer's disease. As Dr. Henneberry's lab has shown, when neuronal ATP levels are compromised by hypoglycemia, hypoxia, inhibition of oxidative phosphorylation or inhibition of $\mathrm{Na}^{+} / \mathrm{K}^{+}$ATPase activity, glutamate is an extremely potent neurotoxin, with an $\mathrm{LD}_{50}$ of about $20 \mu \mathrm{M}$. (Incidentally, Dr. Henneberry's findings address Dr. Gibson's concern that the level of glutamate observed after ischemic insults is insufficient to cause neuronal damage.) These findings are even more intriguing in the light of Dr. Gibson's finding that the activity of 2-oxoglutarate dehydrogenase is reduced by more than $75 \%$ in Alzheimer's brains. Because the enzyme is central to oxidative metabolism as well as to the metabolism of glutamate and aspartate, this dramatic reduction might be expected to decrease neuronal ATP levels and increase glutamate levels. A possible "feed-forward" cycle can be conceived in which reduced levels of 2-oxoglutarate dehydrogenase result in increased glutamate and aspartate levels and decreased oxidative metabolism with resultant decreased ATP levels. This cycle would render the neurons more susceptible to excitotoxic damage and lead to increased intracellular calcium levels which would, in turn, irreversibly inactivate 2-oxoglutarate dehydrogenase. As the neurons die and are lysed by excitotoxic mechanisms, more EAA would be released and cause further excitotoxic damage.

Dr. May notes that glutamate interferes with cystine transport and results in glutathione depletion and subsequent sensitization to free radical damage. This interesting observation suggests yet another mechanism by which EAA may contribute to the pathogenesis of the pathology in Alzheimer's disease. It will be of interest to study the distribution of free radical-scavenging enzymes and enzymes involved in oxidative metabolism in normal brains as well as Alzheimer's disease brains. Such studies may add to our understanding of the selective vulnerability of restricted cell populations as well as the advanced age of onset seen in Alzheimer's disease.

Other EAA-related factors may also help to explain the distribution of the pathology of Alzheimer's disease. As we mentioned in our review, and as Edith McGeer emphasizes, there may be subtypes of NMDA receptors. Furthermore, glycine and PCP modulation of NMDA receptors may not be uniform throughout the brain. Thus, regional variations in the degree of glycinePCP-NMDA coupling could also help to explain the type of selective damage which occurs in Alzheimer's disease. As Dennis Choi and his colleagues have demonstrated, non-NMDA receptors may also mediate excitotoxic neuronal damage. Of course, it is difficult to study such mechanisms in Alzheimer's disease. The development of assays to measure quisqualate receptors linked to ion channels and those linked to inositol triphosphate metabolism $(1,2)$ in postmortem brain should further our understanding of the role of these EAA receptors in Alzheimer's disease. In addition, we have recently described an unique glutamate binding site which is distinct from NMDA, quisqualate or kainate receptors (5). This site is located postsynaptically on neurons, has a submicromolar affinity for glutamate, an unique regional distribution and an interesting pattern of development. The function of this site is unknown and whether it can mediate neurotoxic events remains to be determined. Preliminary experiments indicate that this site is depleted in Alzheimer's disease hippocampal formation. Whether glutamate can still exert excitotoxic effects in the presence of saturating concentrations of both NMDA and quisqualate/kainate antagonists needs to be determined. If so, such effects may be mediated by the recognition site we have identified.

Drs. Gibson and McGeer have correctly pointed out that under certain circumstances, other neurotransmitters, such as dopamine and acetylcholine, may also be neurotoxic. While these neurotransmitters may have direct neurotoxic effects, it is also possible that their toxicity results from an interaction with EAA mechanisms. As we discussed in our review, and as emphasized by Dr. Henneberry, anything which depolarizes neurons, including acetylcholine or dopamine, renders those neurons more vulnerable to excitotoxicity. Perhaps the toxicity produced by these other neurotransmitters could be ameliorated by EAA antagonists. Relevant to this, Schwarcz and Kohler (10) demonstrated cholinergic modulation of kainate toxicity. Brian Meldrum and colleagues have shown that NMDA antagonists protect against pilocarpine-induced limbic seizures (and, presumably, the associated neuronal damage) (7).

Several issues were raised with respect to beta- $\mathrm{N}$-methylaminoL-alanine (BMAA). First, we agree with Dr. McGeer that BMAA is unlikely to activate EAA receptors directly. However, as Weiss and Choi (12) have demonstrated, BMAA in the presence of bicarbonate is excitotoxic and produces excitatory electrophysiological responses. Second, we agree with Dr. Choi that BMAA likely activates NMDA as well as non-NMDA receptors. Third, Dr. McGeer has raised the possibility that the structure of BMAA suggests that it might act at the glycine recognition site. We have examined this issue directly (J. W. McDonald et al., submitted) and can find no evidence for such an interaction.

Therapeutic modulation of excitatory amino acid neurotransmission in Alzheimer's disease might have two goals. First, since there is apparently disruption of EAA neurotransmission in this disease, replacement therapy may be considered. Second. since 
excitotoxic mechanisms may play a role in the pathogenesis of Alzheimer's disease, antagonism of excitotoxicity might be of benefit. In large part, these strategies appear to be mutually exclusive. Dr. Bowen and his colleagues believe that the glycine modulatory site may be an attractive target for therapeutic intervention. This may be true, but which will be better-a glycine agonist to potentially enhance cognition, or a glycine antagonist to retard disease progression? A partial glycine agonist may actually provide the best solution. Because of the convincing evidence that non-NMDA receptors can mediate excitotoxic events, Dr. Choi has suggested that non-NMDA receptor antagonists might have therapeutic benefit. However, non-NMDA EAA receptors probably mediate a large portion of the fast synaptic neurotransmission in the central nervous system, so antagonism of these receptors may have profound deleterious results. As we suggested, it may be possible to exploit EAA agonists as "cognition enhancing" drugs. by combining their use with agents that block specifically the postreceptor mechanisms responsible for toxicity. As Dr. Choi points out, voltage-gated calcium channels may be such a postreceptor target. Finally, as Dr. May proposes, antioxidant agents may reduce EAA toxicity without interfering with normal neurotransmission.

\section{REFERENCES}

1. Cha, J. J.; Greenamyre, J. T.; Nielsen, E. O.; Penney, J. B.; Young, A. B. Properties of quisqualate-sensitive $\mathrm{L}-\left[{ }^{3} \mathrm{H}\right]$ glutamate binding sites in rat brain as determined by quantitative autoradiography. J. Neurochem. 51:469-478; 1988

2. Cha, J. J.; Penney, J. B.; Young, A. B. Ibotenate displaces $\left[{ }^{3} \mathrm{H}\right]$ glutamate from an AMPA-insensitive quisqualate binding site. Soc. Neurosci. Abstr., in press; 1989.

3. Debowey, D. L.; Maragos, W. F.; Hollingsworth, Z.; Greenamyre, J. T.; Young, A. B.; Penney, J. B. Receptor changes in hippocampus of Alzheimer's disease. Soc. Neurosci. Abstr. 13:439; 1987.

4. Greenamyre, J. T.; Penney, J. B.; D'Amato, C. J.; Young, A. B. Dementia of the Alzheimer's type: changes in hippocampal L$\left[{ }^{3} \mathrm{H}\right]$ glutamate binding. J. Neurochem. 48:543-551; 1987.

5. Higgins, D. S.; Greenamyre, J. T.; Cha, J. J.; Penney, J. B.; Young, A. B. A unique glutamate binding site in an autoradiographic assay. Soc. Neurosci. Abstr., in press; 1989.

6. Maragos, W. F.; Chu, D. C. M.; Young, A. B.; D'Amato, C. J.: Penney, J. B. Loss of hippocampal $\left[{ }^{3} \mathrm{H}\right] \mathrm{TCP}$ binding in Alzheimer's disease. Neurosci. Lett. 74:371-376; 1987.

7. Millan, M. H.; Patel, S.; Mello, L. M.; Meldnum, B. S. Focal injection of $\alpha$-amino-7-phosphonoheptanoic acid into prepiriform cortex protects against pilocarpine-induced limbic seizures in rats Neurosci. Lett. 70:69-74; 1986.

8. Monaghan, D. T.; Geddes, J. W.; Yao, D.; Chung, C.; Cotman, C. W. $\left[{ }^{3} \mathrm{H}\right] \mathrm{TCP}$ binding sites in Alzheimer's disease. Neurosci. Lett. 73:197-200; 1987.

9. Mouradian, U. M.; Contreras, P. C.; Monaghan, J. B.; Chase, T. N. $\left[{ }^{3} \mathrm{H}\right] \mathrm{MK}-801$ binding in Alzheimer's disease. Neurosci. Lett. 93: 225-230; 1988.

10. Schwarcz, R.; Kohler, C. Evidence against an exclusive role of glutamate in kainic acid neurotoxicity. Neurosci. Lett. 19:243-249; 1980.

11. Simpson, M. D. C.; Royston, M. C.; Deakin, J. F. W.; Cross, A. J.; Mann, D. M. A.; Slater, P. Regional changes in [ $\left.{ }^{3} \mathrm{H}\right] \mathrm{D}$-aspartate and $\left[{ }^{3} \mathrm{H}\right] \mathrm{TCP}$ binding sites in Alzheimer's disease brains. Brain Res. 462:76-82; 1988

12. Weiss, J. H.; Choi, D. W. Beta-N-methylamino-L-alanine neurotoxicity: requirement for bicarbonate as a cofactor. Science 241:973-975; 1988. 